\title{
A Genetic Analysis of Lysosomal Enzyme Activities in Brahman Cattle
}

\author{
C. P. McPhee and K. G. Reichmann
}

Department of Primary Industries, Animal Research Institute, 665 Fairfield Road, Yeerongpilly, Qld 4105.

\section{Abstract}

Analyses of variance and covariance were carried out on the activities of three lysosomal enzymes in mononuclear blood cells from Brahman cattle. These were hexosaminidase (HEX), beta-D-galactosidase (GAL) and acid alpha-glucosidase (GLU) which had been measured in blood mononuclear cells from 1752 cattle from 6 herds in a Pompe's disease control programme. Herd of origin and date of bleeding significantly affected the level of activity of all enzymes. In addition, HEX and GAL were affected by age and HEX by the sex of the animal bled. Estimates of heritability from sire variances were $0.29 \pm 0.09$ for HEX, 0.31 \pm 0.09 for GAL and $0.44 \pm 0.09$ for GLU. Genetic correlations between all enzymes were positive. The data indicate the existence of a major gene causing Pompe's disease and responsible for $16 \%$ of the genetic variation in GLU. One standard deviation of selection differential for high GLU should almost eliminate Pompe's disease from the population. The efficiency of selection would be aided by estimating the breeding value for GLU using measurements of HEX and GLU and taking account of an animal's sex, age, date of bleeding and herd of origin.

\section{Introduction}

A deficiency of the lysosomal enzyme, acid alpha-glucosidase, is characteristic of the inherited condition, Pompe's disease (generalized glycogenosis type II), which has been described from a wide range of species from man to cat. It has been attributed to a single autosomal gene, recessive for the disease and additive for alpha-glucosidase activity, and has been observed in Australia in both beef Shorthorn and Brahman cattle (Jolly et al. 1977; O'Sullivan et al. 1981). Currently a testing programme operates in Brahman studs to identify genetic carriers of the disease. The test is based on the measurement of acid alpha-glucosidase activity relative to that of two other lysosomal enzymes, betagalactosidase and hexosaminidase in mononuclear blood cells.

This study examines sources of genetic and environmental variation in each of the three enzymes, using data obtained from a number of herds participating in the Pompe's disease control programme. The results are used to develop selection criteria to increase alphaglucosidase activity in the population and thereby reduce the frequency of the gene responsible for Pompe's disease.

\section{Materials and Methods}

The animals included in this study were from Brahman stud herds in central Queensland, some of which had experienced confirmed or suspected cases of Pompe's disease. Blood samples were taken from cattle in these herds between 1986 and 1988. Not all cattle within a herd were sampled on the one occasion. Thus there were a number of sampling dates for each herd.

\section{Enzyme Analysis}

Mononuclear cells were isolated from $20 \mathrm{ml}$ of EDTA blood from each animal, $24 \mathrm{~h}$ after collection. Isoelectric precipitation was used to remove acid alpha-glucosidase from these cells by extracting 
with $0.75 \mathrm{ml}$ of a medium (TKA) containing $0.5 \mathrm{M} \mathrm{KCl}, 0.005 \mathrm{M}$ acetate buffer $(\mathrm{pH} 3.5$ ) and $0.2 \%$ Triton-X100 (Healy 1982). The activities of acid alpha-glucosidase (GLU), beta-D-galactosidase (EC 3.2.1.23) (GAL) and beta-N-acetyl-D-glucosaminidase (EC 3.2.1.30; hexosaminidase) (HEX) were measured in the supernatant of these cell extracts according to Healy (1982), with the exception that a $p$-nitrophenol derivative was used as the substrate for HEX. Activities are expressed as IU $1^{-1}$ of TKA extract.

\section{Data Analysis}

The data analysed were measurements of GLU, HEX and GAL activities in 323 male and 1429 female Brahman cattle from 6 herds. These were the progeny of 199 sires and 1190 dams. Herds were bled on from two to six different occasions, and in all there was a total of 24 different sampling dates across the herds. The average age at bleeding was $48 \pm 0.9$ months.

Data analysis involved the use of least squares to estimate fixed effects and restricted maximum likelihood (Patterson and Thompson 1975) to estimate components of variance. The computer programme used was developed by Robinson et al. (1987). The fixed effects were herd, sampling date, sex and age (covariate). The random effects were sire, dam and residual. An analysis was also carried out on GLU alone using the same model, with HEX and GAL added as covariates.

Dam effects were absorbed and sire effects used to estimate heritabilities and genetic correlations between traits. The sum of cross products for correlation estimates were obtained from the difference between the sire variance of the sum of the traits and the sum of the sire variances obtained for each trait.

Selection indices $(I)$ were constructed by the standard procedure described by Cunningham (1972). These combined HEX, GAL and GLU into a single measurement to predict genetic merit for GLU. These were of the form:

$$
I=b_{1} \cdot \mathrm{HEX}+b_{2} \cdot \mathrm{GAL}+b_{3} \cdot \mathrm{GLU},
$$

where the $b_{n}$ are weighting coefficients.

The efficiencies of the indices as selection criteria for changing GLU were measured as the genetic gain expected in the population per standard deviation change in selection differential. They are also equivalent to the genetic correlations between the index values and the breeding values for GLU.

To search for evidence of major gene effects on the activities of the enzymes, quantile-quantile (Q'-Q) plots were drawn for HEX, GAL and GLU. Hoeschele (1988) demonstrates the use of this technique in detecting the presence of several major genotypic means. In constructing the $Q^{\prime}-\mathrm{Q}$ plots, the ordered sire effects were plotted against cumulative probabilities.

The model given in the Appendix predicts the change in frequency of a gene which contributes a known proportion of the total variation of a continuously variable trait, in this case GLU.

\section{Results}

\section{Fixed Environmental Effects - Herd, Sampling Date, Age, Sex}

\section{Hexosaminidase activity ( $H E X)$}

The average value of HEX was $91 \cdot 2 \pm 0.6 \mathrm{IU}^{-1}$. Males at 94.2 were higher than females at $88 \cdot 2 \mathrm{IU}^{-1}$ (standard error of difference (s.e.d.) 2.3 IU ${ }^{-1}$ ). Age at bleeding had a significant effect on HEX, with values declining at the rate of $0.2 \pm 0.02 \mathrm{IU}^{-1}$ per month. There were considerable herd differences in mean levels of HEX. These ranged from $116 \cdot 3$ to $73.6 \mathrm{IU}^{-1}$ (mean s.e.d., 5.5). Mean HEX values also differed between sampling dates, ranging from $114 \cdot 3$ to $69 \cdot 9 \mathrm{IU}^{-1}$ (mean s.e.d., 6·3).

\section{Beta-D-galactosidase activity (GAL)}

The average value of GAL was $2 \cdot 1 \pm 0 \cdot 02 \mathrm{IU} \mathrm{l}^{-1}$. There was no difference between the sexes but age at bleeding had a significant effect on the level of GAL. This declined $0 \cdot 02 \pm 0.007 \mathrm{IU}^{-1}$ per month of age. Herd averages for GAL ranged from 1.7 to $2 \cdot 8$ IU $1^{-1}$ (mean s.e.d., $0 \cdot 1$ ) and sampling dates from 1.7 to $2.5 \mathrm{IU} \mathrm{l}^{-1}$ (mean s.e.d., $0 \cdot 2$ $\left.\mathrm{IU}^{-1}\right)$.

\section{Alpha-glucosidase activity (GLU)}

The average value of GLU was $0.59 \pm 0.01 \mathrm{IU} \mathrm{l}^{-1}$. Neither sex nor age had any significant effect on GLU. Herd means ranged from 0.49 to $0.76 \mathrm{IU}^{-1}$ (mean s.e.d., 0.06) 
and sampling date means from 0.41 to $0.75 \mathrm{IU} \mathrm{l}^{-1}$ (mean s.e.d., 0.07). The partial regression coefficients of GLU on HEX and GAL were $0.003 \pm 0.0003$ and $0 \cdot 109 \pm 0.012$ IU $1^{-1}$, respectively.

\section{Random Effects - Sire, Dam, Residual}

After removal of the fixed and sire and dam effects on HEX, GAL and GLU, an examination of the distribution of the residuals revealed no significant departure from normality. Probability plots of the distributions of the 199 sire effects on HEX, GAL and GLU are given in Fig. 1. Superimposed on these are the same distributions plotted as histograms.
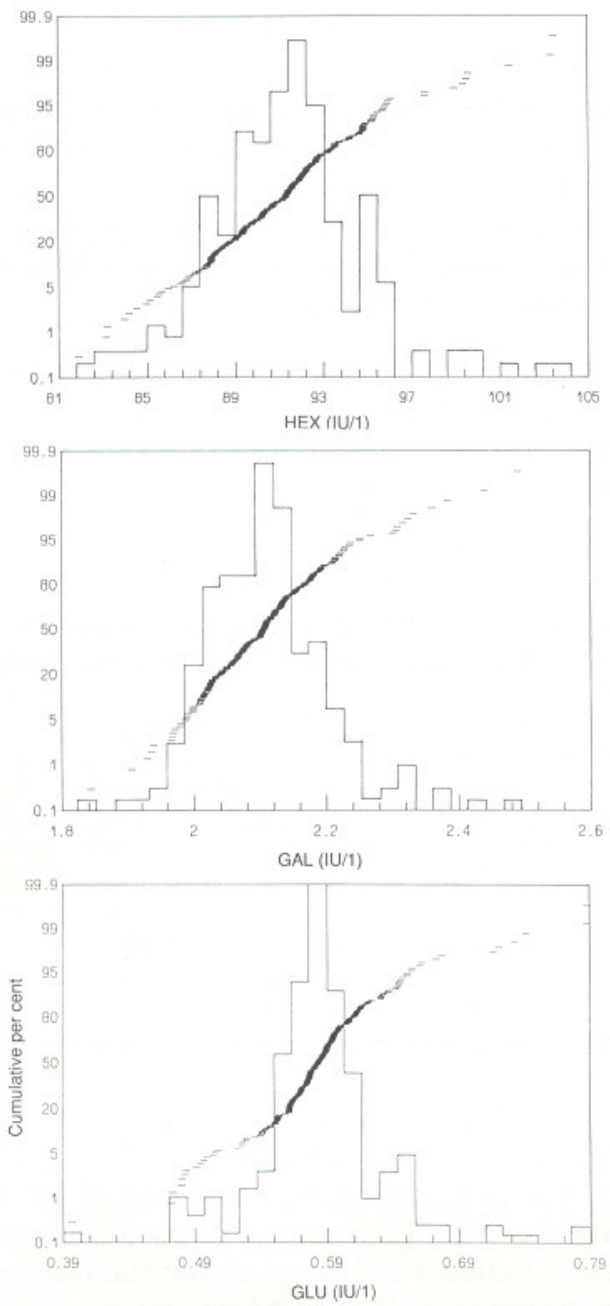

Fig. 1. Quantile-quantile plots of sire effects for the activities of the three enzymes hexosaminidase (HEX), beta-galactosidase (GAL) and alpha-glucosidase (GLU). Histograms of the distributions are superimposed on the probability plots.

Of the three distributions, only that of GLU exhibits a departure from normality. This is revealed by a Kolmogorov statistic of $0 \cdot 12(P<0 \cdot 01)$. The inflections of the $\mathrm{Q}^{\prime}-\mathrm{Q}$ plot and peaks of the histogram for GLU are consistent with a mixture of two normal distributions with means approximating 0.5 and $0.6 \mathrm{IU}^{-1}$ respectively.

\section{Genetic Parameters}

The overall means, standard deviations, heritabilities and genetic and phenotypic correlations of GLU, HEX and GAL are given in Table 1. 
Table 1. Population statistics of the activities of hexosaminidase (HEX), beta-galactosidase (GAL) and acid alpha-glucosidase (GLU)

Heritabilities are underlined on the diagonal. Phenotypic correlations are above and genetic correlations below the diagonal

\begin{tabular}{|c|c|c|c|}
\hline & HEX & GAL & GLU \\
\hline HEX & $0.29+0.09^{\mathrm{A}}$ & $0 \cdot 76$ & $0.52 \pm 0.02$ \\
\hline GAL & $\overline{0.67 \pm 0 \cdot 11}$ & $\underline{0.31+0.09}$ & $0.52 \pm 0.02$ \\
\hline GLU & $0 \cdot 23 \pm 0 \cdot 18$ & $\overline{0.42 \pm 0 \cdot 16}$ & $0.44+0.09$ \\
\hline Mean (IU $\left.1^{-1}\right)$ & $91 \cdot 2 \pm 0 \cdot 6$ & $2 \cdot 1 \pm 0 \cdot 02$ & $\overline{0.59 \pm 0.01}$ \\
\hline s.d. $\left(\mathrm{IU} 1^{-1}\right)$ & $25 \cdot 9$ & 0.67 & 0.27 \\
\hline
\end{tabular}

AStandard error.

The heritabilities of all three enzyme activities are in the intermediate range and do not differ significantly from each other. The heritability of GLU corrected by phenotypic regression on HEX and GAL was $0 \cdot 54 \pm 0 \cdot 12$.

Genetic and phenotypic correlations among the three enzymes activities were positive. Of the genetic correlations, that between HEX and GAL $(0 \cdot 67 \pm 0 \cdot 11)$ was the largest and that between HEX and GLU $(0 \cdot 23 \pm 0 \cdot 18)$ was the smallest.

\section{Selection Indices}

The weighting coefficients $\left(b_{n}\right)$ for a range of selection indices are given in Table 2. Indices 1 to 4 vary in the number of enzymes included. Index 5 includes measurements on all enzyme activities, but its objective is to increase GLU whilst simultaneously preventing change in HEX and GAL.

Table 2. Selection indices with the activity of hexosaminidase (HEX), beta-galactosidase (GAL), and acid alpha-glucosidase (GLU) as measured traits and an increase in GLU as the breeding objective The relative efficiencies of the indices are shown by genetic correlations between the indices and GLU. Responses in HEX, GAL and GLU expected from 1 s.d. of selection are also given

\begin{tabular}{|c|c|c|c|c|c|c|c|}
\hline \multirow{2}{*}{$\begin{array}{l}\text { Index No. } \\
(I)\end{array}$} & \multicolumn{3}{|c|}{ Weightings $\left(b_{n}\right)$} & \multirow{2}{*}{$\begin{array}{l}\text { Genetic } \\
\text { correlation }\end{array}$} & \multicolumn{3}{|c|}{ Response $\left(\mathrm{IU}^{-1}\right)$} \\
\hline & HEX & GAL & GLU & & HEX & GAL & GLU \\
\hline 1 & 0 & 0 & 1 & 0.663 & $2 \cdot 13$ & $0 \cdot 103$ & $0 \cdot 119$ \\
\hline 2 & -0.002 & 0 & $0 \cdot 545$ & 0.712 & -0.744 & 0.062 & $0 \cdot 128$ \\
\hline 3 & 0 & 0.041 & 0.493 & 0.676 & $1 \cdot 17$ & 0.067 & $0 \cdot 121$ \\
\hline 4 & -0.003 & 0.025 & 0.534 & 0.715 & -0.767 & 0.075 & $0 \cdot 128$ \\
\hline 5 & 0.001 & $-0 \cdot 110$ & 0.471 & 0.621 & 0 & 0 & $0 \cdot 111$ \\
\hline
\end{tabular}

As expected, both the correlations between the indices and the breeding value for GLU and the predicted response in GLU rise with the number of enzymes included in the index. Thus, the best selection criterion is Index 4, which includes measurements of all three enzymes. However, Index 2, using only HEX and GLU, is almost as good as Index 4. Compared with the use of GLU alone (Index 1), Indices 4 and 2 are 1.08 times as efficient as selection criteria. Using Index 5 which restricts change in HEX and GAL, sacrifices $13 \%$ of the gain possible in GLU by using Index 4 .

Predicted responses in GAL vary according to the index used, but are in the same direction as GLU. Responses in HEX vary in direction, being negative for those indices in which HEX has a negative weighting. The use of GLU corrected by phenotypic regression on HEX and GAL, had a heritability of $0.54 \pm 0 \cdot 11$ and a phenotypic standard deviation of $0.22 \mathrm{IU} \mathrm{l}^{-1}$. Using this as the selection criterion would give a response from 1 s.d. of selection of $0 \cdot 121$, the same as Index 3 and only $2 \%$ more than using GLU alone. 


\section{Discussion}

A number of sources of variation and covariation in the activities of the three enzymes were identified. The environmental effects of sampling date and herd caused variation in the activities of all three enzymes. Levels of HEX and GAL declined with age, and males had higher HEX values than females. In estimating breeding values for GLU, the enzyme of prime interest, some account should be taken of these environmental effects, e.g. by using deviations from herd by sampling date means rather than absolute values.

The objective of selection to increase GLU is, of course, to reduce the incidence of Pompe's disease. As shown in the Appendix 1, the extent to which this can be achieved depends on the contribution of the gene causing the condition to the total genetic variation in GLU and on the frequency of this gene. Although not evident in the distribution of individual animal phenotypic measurements of GLU, the distribution of sire effects obtained from the analysis (Fig. 1), is suggestive of bimodality. Given that this is due to the gene, which when homozygous causes Pompe's disease, the two means estimated at 0.5 and $0.6 \mathrm{IU} \mathrm{l}^{-1}$ would then represent the two types of progeny, the one produced by heterozygous sires, and the other by homozygous normal sires. Assuming both types of sire had a random allocation of mates with respect to the gene, a value of $0 \cdot 2$, twice the difference in means, is considered to be a reasonable estimate of $a$, the gene's effect in this population, assuming additivity.

Quite high levels of genetic variation were found in the activities of all three enzymes, heritabilities ranging from 0.29 for HEX to 0.44 for GLU. This indicates that all three should respond readily to selection. The genetic correlations between the activities of the enzymes were of sufficient magnitude for measurements of all to be considered in predicting the breeding value of any one. This was demonstrated by the construction of the selection indices, although the advantage of using these in place of GLU itself does not exceed $8 \%$, owing to the high heritability of GLU. The measurement of HEX makes a more useful contribution than GAL to the prediction of an animal's breeding value for GLU, but this contribution would have to be weighed against the cost of determining HEX levels. An increase in GLU by selection will result in changes in HEX and GAL, the magnitude and direction of these changes depending on the index used (Table 2). If these changes were judged to be undesirable, the restricted Index 5 could be used as a selection criterion. At the cost of a $13 \%$ reduction in the maximum possible selection response in GLU, the levels of both HEX and GAL could be held stationary by the use of this index. The phenotypic regression coefficients of GLU on HEX and GAL were very close to those reported by Reichmann et al. (1987) for a herd of Friesian cattle known to be free of Pompe's disease. As expected from genetic theory, correcting GLU by phenotypic regression on HEX and GAL gave a selection criterion inferior to the best index, Index 4, which was formed by using genetic regressions.

An alternative method of estimating $a$ would have been to use an individual animal rather than a sire model to obtain the distributions, then $a$ would have equalled the difference in mean of the distributions. This approach was not taken because individual breeding values would have been of lower reliability than sire values and may have been biased by maternal effects.

Data from this project and from field observations suggest that approximately 5 in 1000 Brahman calves born in central Queensland exhibit Pompe's disease. Assuming random mating of their parents, this gives an estimate of 0.07 for the frequency $(q)$ of the gene and 0.13 for the frequency of heterozygotes. The latter value is consistent with the distribution of GLU in Fig. 1, where $6 \cdot 5 \%(50 \%$ of $0 \cdot 13)$ of the sire effects are less than $0 \cdot 5$, the apparent mean of heterozygous sire effects. From Appendix 1, the genetic variance in GLU attributable to the gene is calculated at $0.005 \mathrm{IU}^{-1}$, given that $q$ is 0.07 and $a$ is 0.2 . This variance is only $16 \%$ of the total of $0.032 \mathrm{IU} \mathrm{I}^{-1}$ calculated for the trait from sire variances, the remaining $84 \%$ presumably being due to other genes whose individual effects cannot be detected. 
It follows that $1 \mathrm{s.d}$. of selection differential should increase the level of GLU in the population by $0 \cdot 12 \mathrm{IU}^{-1}$ (Table 2), but only $16 \%$ of this change would be due to a reduction in the frequency of the gene causing Pompe's disease, the remainder being due to other genes.

The low estimate of the gene's contribution to variation in GLU is surprising. Disassortative mating of the parents of the animals analysed would have led to an underestimate of $q$, and hence its contribution to variation in GLU, but this seems unlikely since the enzyme activities of most of the parents would not have been known at mating. Even allowing for the possibility of downward bias, there is clear evidence for the existence of minor genes which would lead to low values of GLU in some families, even in the absence of the Pompe's disease gene.

Table 3. Change in the frequency of the gene $\boldsymbol{n}$ due to lethality and one standard deviation of truncation selection on the continuous scale of the selection criterion

The effect of the gene is measured in phenotypic standard deviations $\left(a P^{-1 / 2}\right)$

\begin{tabular}{llccc}
\hline $\begin{array}{l}\text { Initial } \\
\text { frequency } \\
(q)\end{array}$ & $a P^{-1 / 2}$ & $\begin{array}{c}\text { Frequency change } \\
\text { Selection }\end{array}$ & $\begin{array}{c}\text { Lethality } \\
(\mathrm{d} q)\end{array}$ & Total \\
\hline 0.05 & 0.5 & -0.022 & -0.002 & -0.024 \\
0.05 & 0.75 & -0.032 & -0.002 & -0.034 \\
0.05 & 1.0 & -0.043 & -0.002 & -0.045 \\
$0 \cdot 10$ & 0.5 & -0.037 & -0.009 & -0.046 \\
$0 \cdot 10$ & 0.75 & -0.056 & -0.009 & -0.065 \\
$0 \cdot 10$ & 1.0 & -0.074 & -0.009 & -0.083 \\
$0 \cdot 15$ & 0.5 & -0.048 & -0.020 & -0.068 \\
0.15 & 0.75 & -0.072 & -0.020 & -0.092 \\
0.15 & 1.0 & -0.096 & -0.020 & -0.116 \\
\hline
\end{tabular}

Applying the arguments set out in the Appendix, 1 s.d. of selection differential for increased GLU is expected to result in a decline of approximately 0.05 in $q$ (Table 3 ). Under random mating this would lead to a fall in the incidence of Pompe's disease over the same period to 5 in 10000 calves born. At the existing level of culling of $0 \cdot 15$ in the present Pompe's disease control programme, it should take 3.5 generations to accomplish this. The recommended selection criterion would be an estimate of the breeding value for GLU using measurements of HEX and GLU and taking account of an animal's sex, age, date of bleeding and herd of origin.

\section{Acknowledgments}

Thanks are due to staff of the Biochemistry Branch and to Dr F. C. Baldock and $\mathrm{Mr}$ G. M. Macbeth of the Animal Research Institute for assistance with data preparation.

\section{References}

Cunningham, E. P. (1972). Theory and application of statistical selection methods. Proc. XIV Br. Poultry Breeder's Roundtable, Birmingham, pp. 1-38.

Healy, P. J. (1982). Diagnosis of genotype for generalised glycogenosis in cattle. Biochem. Med. 28 , 224-8.

Hoeschele, I. (1988). Statistical techniques for detection of major genes in animal breeding data. Theor. Appl. Genet. 76, 311-19.

Jolly, R. D., Van-de-Water, N. S., Richards, R. B., and Dorling, P. R. (1977). Generalised glycogenesis in beef Shorthorn cattle - heterozygote detection. Aust. J. Exp. Biol. Med. Sci. 55, 141-50.

O’Sullivan, B. M., Healy, P. J., Fraser, I. R., Nieper, R. E., Whittle, R. J., and Sewell, C. A. (1981). Generalised glycogenosis in Brahman cattle. Aust. Vet. J. 57, 227-9. 
Patterson, H. D., and Thompson, R. (1975). Maximum likelihood estimation of components of variance. Proc. 8th Int. Biometric Conf., pp. 197-207.

Reichmann, K. G., Twist, J. O., McKenzie, R. A., and Rowan, K. J. (1987). Inhibition of bovine alpha-glucosidase by Castanospermum australe and its effect on the biochemical identification of heterozygotes for generalised glycogenosis type II (Pompe's disease) in cattle. Aust. Vet. J. 64, 274-6.

Robinson, D. L., Digby, P. G. N., Williams, E. R., and Ratcliff, D. (1987). Estimation of variance components in non-orthogonal data by residual maximum likelihood. REML user manual. Scottish Agricultural Statistics Service, University of Edinburgh.

Manuscript received 15 May 1989, accepted 1 August 1989

\section{Appendix 1. The effect of truncation selection on the frequency of a major gene}

Let $q$ be the frequency of a gene $n$ in breeding animals. It has an additive effect on a normally distributed trait and is recessive lethal for fitness. It is desired to approximate the change in frequency of $n$ through one generation of truncation selection on the trait.

With random mating of the parents, the following describes their progeny just before selection as parents of the following generation:

$\begin{array}{llll}\text { Genotype } & N N & N n & n n \\ \text { Fitness } & 1 & 1 & 0 \\ \text { Frequency of genotype } & x & y & 0 \\ \text { Effect on trait }(e) & a & 0 & - \\ \text { Frequency of the } n \text { gene }(q) & 0 & 0 \cdot 5 & -\end{array}$

where $x=(1-q)(1+q)^{-1}$ and $y=2 q(1+q)^{-1}, C$ is the covariance between $q$ and $e$ and $C=-\operatorname{axy} 2^{-1}$

$P$ is the phenotypic variance of the trait, and

$$
P=G+E,
$$

where $G$ is the genetic variance due to the locus and

$$
G=a^{2} x(1-x) \text {. }
$$

$E$ is the residual variance due to environmental effects and the effect of genes at other loci.

$b$ is the regression of $q$ on phenotype and:

$$
b=C P^{-1} \text {. }
$$

$\mathrm{d} q$ is the change in $q$ resulting from $i$ standard deviations of truncation selection on the trait and

or

$$
\begin{aligned}
& \mathrm{d} q=-i b P^{1 / 2} \\
& \mathrm{~d} q=-i C P^{-1 / 2} .
\end{aligned}
$$

To this change in the frequency of $n$ due to selection on the metric scale must be added the change due to the lethality of $n n$ genotypes given by:

$$
\mathrm{d} q^{\prime}=-q^{2}(1+q)
$$

Using these formulae, the change in gene frequency per generation for each standard deviation of selection was calculated for a range of values of $q$ and $a P^{-1 / 2}$, the effect of the $n$ gene in phenotypic standard deviations of the selection criterion. The results are given in Table 3. 